\title{
Máster en Ciencia de Datos e Ingeniería de Computadores: una apuesta por la formación especializada en el sector de las TIC
}

\author{
Fernando Rojas ${ }^{1}$, Andrés Cano $^{2}$, Manuel Gómez ${ }^{2}$, Julio Ortega ${ }^{1}$, Francisco \\ Herrera $^{2}$, Rocío Romero-Zaliz², Jesús González ${ }^{1}$ \\ ${ }^{1}$ Departamento de Arquitectura y Tecnología de Computadores. ETSI Informática y de \\ Telecomunicación. Universidad de Granada. \\ \{frojas,jortega, jesusgonzalez\}@ugr.es \\ ${ }^{2}$ Departamento de Ciencias de la Computación e Inteligencia Artificial. ETSI Informática y \\ de Telecomunicación. Universidad de Granada. \\ \{acu, mgomez, herrera, rocio\}@decsai.ugr.es
}

\begin{abstract}
Resumen. En el curso 2014-2015 se ha impartido por vez primera el "Máster en Ciencia de Datos e Ingeniería de Computadores" en la Universidad de Granada. Este máster surge de la unión de los anteriores "Máster en Soft Computing y Sistemas Inteligentes" y "Máster en Ingeniería de Computadores y Redes", renovando completamente sus contenidos y ofreciendo una oportunidad de asociación entre varios grupos de investigación con un enorme potencial en el ámbito de las Tecnologías de la Información y de las Comunicaciones (TIC). Este máster proporciona al estudiante la oportunidad de formarse profesionalmente en campos con una gran demanda de personal cualificado y de enorme interés social, tales como el análisis de grandes cantidades de datos (big data) o el desarrollo de sistemas de propósito específico y plataformas de computación de altas prestaciones.
\end{abstract}

Palabras Clave: Ciencia de datos, ingeniería de computadores, Espacio Europeo de Educación Superior (EEES), máster, big data, computación de altas prestaciones, sistemas específicos.

\begin{abstract}
The Master's Degree in Data Science and Computer Engineering has been taught at the University of Granada for the first time during academic year 2014-2015. This master comes from the combination of former masters in Soft Computing and Computer Engineering, but it completely renovates its contents and it provides an opportunity for partnership and collaboration between several research groups with a great potential in the field of Information Technology and Communications (ICT). The proposed Master's Degree trains students as researchers in areas with a high demand for qualified staff and huge social interest, such as the analysis of large amounts of data (big data) or the development of embedded systems and platforms for high-performance computing.
\end{abstract}

Keywords: Data science, computer engineering, European Higher Education Area (EHEA), Master's Degree, embedded systems, high-performance computing. 


\section{Introducción}

\subsection{Motivación}

Los estudios de un máster tienen como principal finalidad que el estudiante adquiera una formación avanzada, ya sea de carácter especializado o multidisciplinar. Éste es el escenario en que se propone el Máster Universitario Oficial en Ciencia de Datos e Ingeniería de Computadores por la Universidad de Granada, siguiendo la disposición de los Reales Decretos 1393/2007 y 861/2010 por los cuales se establece que los estudios de máster universitario de investigación constituyen el período formativo previo al de doctorado.

El Máster Universitario Oficial en Ciencia de Datos e Ingeniería de Computadores se presenta como una oportunidad de sinergia entre varios grupos de investigación en temas de gran demanda profesional y científica, que sin ninguna duda, ya forma parte del perfil profesional demandado en la actualidad en el ámbito de las Tecnologías de la Información y de las Comunicaciones. Por una parte, la necesidad de analizar grandes volúmenes de datos hacen de la Ciencia de Datos una de las disciplinas que mayor demanda de empleo generará en el futuro inmediato en todo el mundo. Por otra parte, el ingente volumen de datos a tratar precisa de plataformas de cómputo de altas prestaciones y sistemas específicos en el ámbito de la Ingeniería de Computadores.

Este máster proporciona al estudiante la oportunidad de formarse profesionalmente en estas líneas a través de un personal docente integrado en diferentes grupos de investigación de reconocido prestigio internacional. Se presenta como un título único que integra los requerimientos de cómputo de altas prestaciones y de sistemas de uso específico ante la necesidad de obtener información útil a partir de grandes cantidades de datos. Se pueden encontrar todos los detalles sobre este título en la página web del máster [1] y, de forma resumida, en el tríptico del máster (se reproduce la portada en la Figura 1), disponible en formato digital en la misma dirección URL.

\subsection{Antecedentes}

El Máster Universitario Oficial en Ciencia de Datos e Ingeniería de Computadores se propone como un nuevo máster que ha surgido de la unión de los anteriores "Máster en Soft Computing y Sistemas Inteligentes" y "Máster en Ingeniería de Computadores y Redes".

El Máster en Soft Computing y Sistemas Inteligentes surgió como la evolución natural del Programa de Doctorado "Diseño, Análisis y Aplicaciones de Sistemas Inteligentes" al Espacio Europeo de Educación Superior (EEES). Dicho programa obtuvo la Mención de Calidad del Ministerio de Educación y Ciencia desde el año 2003 (Mención de Calidad MCD2003-00509) hasta el 2006, año en el que se sustituye por el Posgrado en Ciencias de la Computación y Tecnología Informática, obteniendo la mención de calidad durante su impartición (MCD2007-00212). 


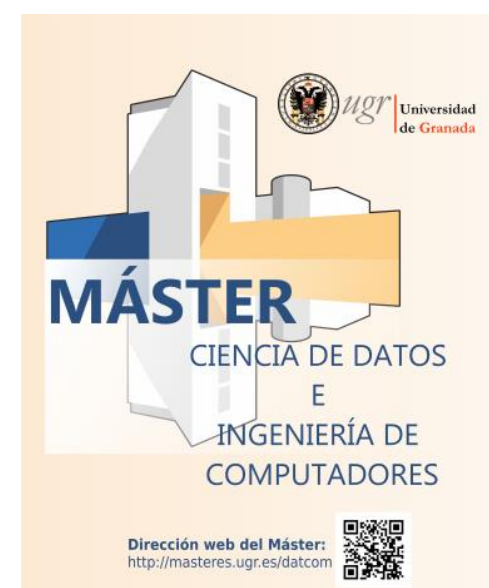

Figura 1. Portada del tríptico informativo del Máster en Ciencia de Computadores e Ingeniería de Computadores (disponible en http://masteres.ugr.es/datcom/).

El Máster Universitario en Ingeniería de Computadores y Redes surgió igualmente a partir del proceso de adaptación al EEES (R.D. 56/2005) del Programa de Doctorado "Ingeniería de Computadores: Perspectivas y Aplicaciones". Este Programa de Doctorado se impartió ininterrumpidamente desde el curso 1999-2000 por el departamento de Arquitectura y Tecnología de Computadores de la Universidad de Granada, integrado por profesores de las áreas de Arquitectura y Tecnología de Computadores e Ingeniería de Sistemas y Automática, todos ellos miembros del grupo de investigación CASIP (Circuitos y Sistemas para el Procesamiento de la Información), reconocido como grupo de excelencia de la Junta de Andalucía con la referencia TIC-117. El programa obtuvo la Mención de Calidad del Ministerio (MCD2004-00438, BOE 5/7/2004). Desde ese momento, el programa ha concurrido a todas las convocatorias que se han sucedido para la renovación y seguimiento, habiéndose mantenido la Mención de Calidad desde 2004. Actualmente, estos dos programas de doctorado se encuentran en proceso de extinción, siendo sustituidos por el Programa Oficial de Doctorado en Tecnologías de la Información y la Comunicación de la Universidad de Granada [2].

\subsection{Proceso de verificación}

La solicitud de verificación del actual Máster en Ciencia de Datos e Ingeniería de Computadores se realizó a través de la Universidad de Granada, aprobada por Consejo de Gobierno en su reunión de 8 de abril de 2014. En [3] se puede consultar el documento de solicitud de verificación completo. En respuesta, se recibió el informe favorable por parte de la Dirección de Evaluación y Acreditación de la Agencia Andaluza del Conocimiento (DEVA) para la verificación del título oficial en su reunión celebrada en Córdoba el 30 de junio de 2014. La Consejería de Economía, Innovación, Ciencia y Empleo de la Junta de Andalucía a través del Decreto $113 / 2014$, de 15 de julio, por el que se autoriza la implantación de enseñanzas universitarias de Grado, Máster y Doctorado de las Universidades públicas andaluzas 
para el curso 2014/2015 recoge, entre los títulos de máster que se van a impartir, al Máster en Ciencia de Datos e Ingeniería de Computadores [4]. La resolución de 2 de octubre de 2014 de la Secretaría General de Universidades publica el Acuerdo del Consejo de Ministros de 26 de septiembre de 2014 por el que se establece el carácter oficial de determinados títulos de Máster (entre los cuales figura el que aquí se describe) y su inscripción en el Registro de Universidades, Centros y Títulos [5]. Finalmente, la resolución de 25 de noviembre de 2014 de la Universidad de Granada publicada en [6] publica el plan de estudios del Máster en Ciencia de Datos e Ingeniería de Computadores.

\subsection{Diferenciación con títulos similares en la Universidad de Granada}

La Universidad de Granada incluye entre su oferta actual varios títulos de Máster en el ámbito de las Tecnologías de la Información y Comunicación. En cualquier caso, el Máster en Ciencia de Datos e Ingeniería de Computadores se diferencia notablemente de todos ellos en cuanto a su justificación, competencias y plan de estudios.

En primer lugar, se ofrece un máster universitario que se imparte en la ETS de Ingeniería Informática y Telecomunicación denominado "Máster en Desarrollo de Software" [7]. Este máster se integra en el Programa Oficial de Doctorado en Tecnologías de la Información y la Comunicación. No obstante, sus contenidos son completamente diferentes a los propuestos en este máster, centrándose en tareas de desarrollo e ingeniería del software e investigación en la interacción personaordenador.

Adicionalmente se oferta el máster de orientación profesional "Máster en Gestión y Tecnologías de Procesos de Negocio" [8] que podría incluirse en el ámbito de las TIC, pero que se diferencia claramente del descrito aquí, debido a su orientación específica a la gestión empresarial y las tecnologías asociadas.

Finalmente, la Universidad de Granada ha puesto en marcha los Másteres Universitarios que habilitan para el ejercicio de la profesión de Ingeniero de Telecomunicación [9] y de Ingeniero en Informática [10]. Estos másteres proporcionan competencias profesionales reguladas y no tiene un perfil marcado en un área concreta de las tecnologías de la información y las comunicaciones.

\section{Justificación e interés del máster para la sociedad}

El Máster en Ciencia de Datos e Ingeniería de Computadores ofrece un valor diferenciador a través de las posibilidades de obtener una formación interdisciplinar y una óptima propuesta para la inserción laboral e investigadora, actualizada e incorporando líneas de investigación y transferencia muy significativas en el ámbito profesional de las Tecnologías de la Información y la Comunicación.

Todos los alumnos que cursen este máster obtienen un título oficial con reconocimiento en todo el Espacio Europeo de Educación Superior (EEES), y además les permite acceder a la realización de la tesis doctoral dentro del Programa de Doctorado Tecnologías de la Información y las Comunicaciones [2], siempre que se cumplan las condiciones que exige la normativa vigente. 
La demanda laboral de especialistas en ciencia de datos e ingeniería de computadores hace de este perfil informático uno de los más solicitados por parte de todo tipo de empresas tanto a nivel nacional como internacional. El sector de las TIC es uno de los pocos que puede presumir de tener paro cero y las expectativas son aún mejores, asegurándose desde el Colegio de Ingenieros Informáticos de España que "en los próximos cinco años el mercado demandará medio millón de puestos de trabajo relacionados con la economía digital" [11]. Si se restringe el ámbito de las TIC a la ciencia de datos e ingeniería de computadores, las perspectivas son mucho mejores: "El 'big data' arrasa en las escuelas de negocios" [12], "El cientifico de datos: una novedosa y necesaria profesión" [13], etc.

En el ámbito local, en palabras del propio Director de la ETS de Ingenierías Informática y de Telecomunicación, Joaquín Fernández Valdivia, las TIC constituyen el nuevo motor económico de la ciudad de Granada, constituyendo lo que denomina una "isla tecnológica" en España en la que confluyen la excelencia en formación, investigación y transferencia para ofrecer un "ecosistema único a través del cual es posible brindar verdadero apoyo a buenas ideas y prometedores proyectos de alto contenido tecnológico al disponer de una abundante oferta de profesionales universitarios de altísima preparación tecnológica unida a una proliferación de vocaciones empresariales" [14].

La calidad de la formación que se va a proporcionar en este máster está avalada por la excelencia de la Universidad de Granada en el campo de la Informática (Computer Science). Existen dos rankings o clasificaciones de calidad de las universidades de reconocido prestigio internacional que se describen: el ranking de Shanghai (Academic Ranking of World Universities, ARWU) [15] y el ranking de Taiwan (National Taiwan University Ranking, NTU Ranking) [16]. En la edición de 2014 del ranking de Shanghai, la Universidad de Granada ocupa el puesto 43 del mundo en el campo de la Informática (Computer Science), siendo la primera universidad española y la séptima de Europa. En cuanto a la clasificación elaborada por la National Taiwan University a partir de los datos de publicaciones científicas de las bases de datos Web of Science de Thomson Reuters, la Universidad de Granada se sitúa también como la primera universidad española en Informática y en la posición $20^{\mathrm{a}}$ del mundo (Figura 2) y $3^{\text {a }}$ de Europa. Las siguientes universidades españolas en esta clasificación son la Universidad Politécnica de Cataluña, en el puesto 46, y la Universidad Politécnica de Valencia en el 77.

\section{Estructura del Máster}

\subsection{Especialidades que incluye el Máster}

El máster propuesto incluye dos especialidades que se corresponden, en cierta medida, a los anteriores másteres de Soft Computing y Sistemas Inteligentes y el de Ingeniería de Computadores y Redes. El estudiante puede elegir una especialidad de entre las dos ofertadas, o bien realizar una configuración multidisciplinar en su perfil de optatividad. Las especialidades propuestas se describen a continuación. 


\begin{tabular}{|c|c|c|}
\hline World Rank & University & Total Score details \\
\hline 1 & Nanyang Technological University & 89.5 \\
\hline 2 & Massachusetts Institute of Technology & 87.6 \\
\hline 3 & University of California- Berkeley & 85.8 \\
\hline 3 & Stanford University & 85.8 \\
\hline 5 & Tsinghua University & 81.2 \\
\hline 6 & National University of Singapore & 79.0 \\
\hline 7 & Harvard University & 78.8 \\
\hline 8 & University of California- San Diego & 78.1 \\
\hline 9 & City University of Hong Kong & 77.6 \\
\hline 10 & University of Illinois- Urbana-Champaign & 77.2 \\
\hline 11 & Carnegie Mellon University & 76.7 \\
\hline 12 & Swiss Federal Institute of Technology - Zurich & 74.5 \\
\hline 13 & Zhejiang University & 74.4 \\
\hline 14 & Harbin Institute of Technology & 73.2 \\
\hline 15 & Hong Kong Polytechnic University & 73.0 \\
\hline 16 & Georgia Institute of Technology & 72.2 \\
\hline 17 & Shanghai Jiao Tong University & 72.1 \\
\hline 18 & University of Michigan- Ann Arbor & 70.1 \\
\hline 19 & Swiss Federal Institute of Technology - Lausanne & 69.8 \\
\hline 20 & University of Granada & 69.7 \\
\hline 21 & The University of Texas- Austin & 69.6 \\
\hline 22 & University of Southern California & 69.5 \\
\hline 23 & University of Maryland- College Park & 68.0 \\
\hline 23 & University of Oxford & 68.0 \\
\hline 25 & Huazhong University of Science \& Technology & 67.4 \\
\hline
\end{tabular}

Figura 2. 'Top 25' de Universidades a nivel mundial en el campo de la Informática (Computer Science), de acuerdo con el 'Ranking de Taiwán 2014'.

(http://nturanking.lis.ntu.edu.tw/DataPage/TOP300.aspx?query=ComputerScience\&y=2014)

- Especialidad en Ingeniería de Computadores y Redes. Las perspectivas en la ingeniería de computadores y las redes están determinadas tanto por las posibilidades que ofrecen las mejoras tecnológicas como por los condicionantes que establecen las aplicaciones dominantes en el mercado: se trata de disponer de computadores que aprovechen la tecnología de forma óptima, ejecutando eficientemente las aplicaciones (fundamentalmente las más demandadas), y ajustándose a las restricciones que impone el mercado. El hecho de disponer de un cada vez mayor número de datos y la necesidad de obtener información útil a partir de dichos datos en un tiempo razonable implica la demanda imprescindible de plataformas de cómputo de altas prestaciones (HPC, High performance Computing) [17] y sistemas de propósito específico que se adapten al entorno de uso tanto a nivel de hardware como de software $[18,19]$. 
- Especialidad en Ciencia de Datos y Tecnologías Inteligentes. Big data y la ciencia de datos utilizan algoritmos y tecnología de estadística de datos para procesar grandes cantidades de datos con el objetivo de extraer conocimiento [20-24]. Se aplican a áreas tan diversas como la genómica, física, energía, transportes, finanzas, marketing, medicina, educación, etc. Todas las previsiones indican que su aplicación crecerá de forma notable en los próximos años, ya que el proceso de recogida de datos mediante ordenadores, dispositivos inteligentes, sensores y web, entre otros, aumenta de forma notable. Ante esta avalancha de datos, hay una percepción creciente de la necesidad de analizarlos, ordenarlos y visualizarlos en busca de relaciones, patrones y agrupaciones que no pueden obtenerse mediante su simple observación. En definitiva, todo apunta a que habrá una gran demanda de profesionales dedicados a esta tarea en los próximos años.

Las dos especialidades propuestas se complementan mutuamente, ya que el tratamiento de grandes masas de datos sólo es posible con una adecuada infraestructura de cómputo. Esto hace que los equipos profesionales de trabajo en ciencia de datos e ingeniería de computadores tengan que conformarse con personal cualificado en ambos perfiles de conocimiento. De esta forma, se justifica la propuesta de ambas especialidades de forma conjunta y coordinada en este máster.

\subsection{Propuesta formativa}

Para obtener el título de Máster se han de superar 60 créditos ECTS de entre un total de 128 ECTS ofertados. La estructura del máster es la siguiente (Figura 3):

- Asignaturas obligatorias: 12 créditos repartidos en 3 asignaturas de 4 ECTS que incluyen materias comunes relacionadas con la metodología de la investigación en TIC, técnicas de investigación, análisis de datos, oportunidades de innovación, etc.

- Asignaturas de nivelación de conocimientos: se ofertan 16 créditos de los que el estudiante cursará 2 asignaturas (8 ECTS) para complementar su formación y atender a las diferentes necesidades para el acceso al bloque de especialización con suficientes garantías.

- Asignaturas optativas: el alumno debe cursar 28 créditos de optatividad. El estudiante podrá elegir una especialidad de entre las 2 planteadas o bien realizar una configuración multidisciplinar en su perfil de optatividad. Para obtener la distinción de una especialidad dentro del título de máster el estudiante deberá haber superado al menos 24 créditos dentro de la especialidad correspondiente. El resto de créditos (4) se pueden elegir libremente de entre todos los ECTS optativos. Se ofrecen 44 ECTS por especialidad, para un total de 88 ECTS ofertados.

- Trabajo Fin de Máster: el máster concluirá con la elaboración y defensa de un Trabajo de Fin de Máster de 12 ECTS que deberá estar orientado a la evaluación de competencias asociadas al título. 


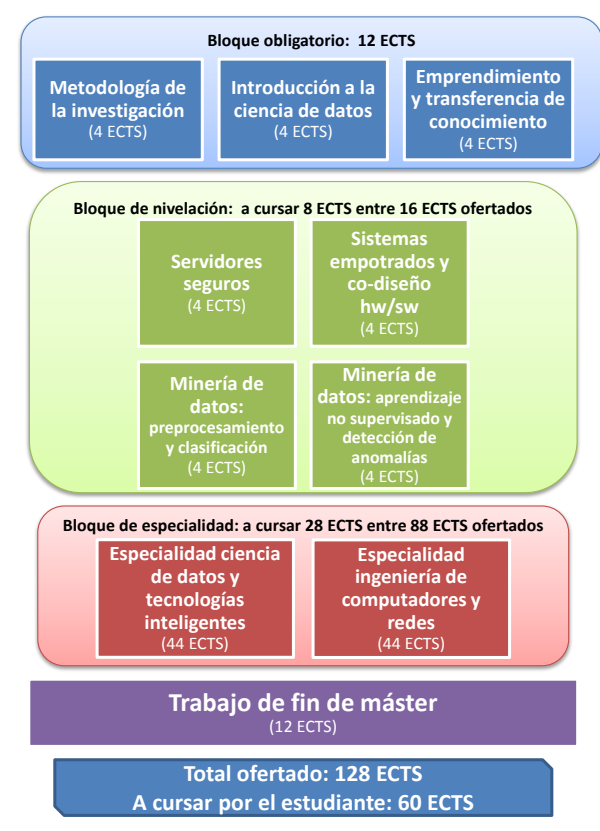

Figura 3. Resumen de la Propuesta formativa del Máster en Ciencia de Datos e Ingeniería de Computadores

\subsection{Descripción detallada del plan de estudios}

Las Tablas 1, 2 y 3 detallan los nombres y asignación de créditos ECTS de las asignaturas que componen cada uno de los bloques descritos en la Figura 3.

Tabla 1. Materias que componen los módulos comunes (obligatorio, nivelación y TFM)

\begin{tabular}{|l|l|c|}
\hline Módulo & Materias & ECTS \\
\hline \multirow{4}{*}{ Módulo Obligatorio } & Metodología de la investigación & 4 \\
\cline { 2 - 3 } & Introducción a la ciencia de datos & 4 \\
\cline { 2 - 3 } & $\begin{array}{l}\text { Emprendimiento y transferencia de } \\
\text { conocimiento }\end{array}$ & 4 \\
\hline \multirow{4}{*}{$\begin{array}{l}\text { Módulo de Nivelación de } \\
\text { Conocimientos }\end{array}$} & Servidores seguros & 4 \\
\cline { 2 - 3 } & Sistemas empotrados y co-diseño hw/sw & 4 \\
\cline { 2 - 3 } & $\begin{array}{l}\text { Minería de datos: preprocesamiento y } \\
\text { clasificación }\end{array}$ & 4 \\
\cline { 2 - 3 } & $\begin{array}{l}\text { Minería de datos: aprendizaje no } \\
\text { supervisado y detección de anomalías }\end{array}$ & 4 \\
\hline Trabajo Fin de Máster & Trabajo fin de máster & 12 \\
\hline
\end{tabular}


Tabla 2. Materias que componen la especialidad "Ingeniería de Computadores y Redes"

\begin{tabular}{|c|c|c|c|}
\hline Especialidad & Módulo & Materias & ECTS \\
\hline \multirow{11}{*}{$\begin{array}{l}\text { Especialidad en } \\
\text { Ingeniería de } \\
\text { Computadores } \\
\text { y Redes }\end{array}$} & \multirow{5}{*}{$\begin{array}{l}\text { Computación } \\
\text { de Altas } \\
\text { Prestaciones }\end{array}$} & $\begin{array}{l}\text { Computación de altas prestaciones para } \\
\text { clasificación y optimización }\end{array}$ & 4 \\
\hline & & $\begin{array}{l}\text { Biología computacional con big data-omics e } \\
\text { ingeniería biomédica }\end{array}$ & 4 \\
\hline & & Ingeniería de servidores web & 4 \\
\hline & & $\begin{array}{l}\text { Modelado de sistemas y predicción de series } \\
\text { temporales }\end{array}$ & 4 \\
\hline & & $\begin{array}{l}\text { Procesamiento de la señal de altas prestaciones } \\
\text { en biomedicina }\end{array}$ & 4 \\
\hline & \multirow{6}{*}{$\begin{array}{l}\text { Sistemas de } \\
\text { Aplicación } \\
\text { Específica }\end{array}$} & Internet de las cosas & 4 \\
\hline & & Arquitecturas de altas prestaciones para visión & 4 \\
\hline & & Mecatrónica y sistemas aero-espaciales & 4 \\
\hline & & Neurociencia computacional y neuroingeniería & 4 \\
\hline & & Sistemas de visión bioinspirados & 4 \\
\hline & & Robótica móvil y neurobótica & 4 \\
\hline
\end{tabular}

Tabla 3. Materias que componen la especialidad "Ciencia de Datos y Tecnologías Inteligentes"

\begin{tabular}{|c|c|c|c|}
\hline Especialidad & Módulo & Materias & ECTS \\
\hline \multirow{11}{*}{$\begin{array}{l}\text { Especialidad en } \\
\text { Ciencia de } \\
\text { Datos y } \\
\text { Tecnologías } \\
\text { Inteligentes }\end{array}$} & \multirow{6}{*}{$\begin{array}{l}\text { Modelos } \\
\text { avanzados de } \\
\text { ciencia de } \\
\text { datos }\end{array}$} & Modelos gráficos probabilísticos & 4 \\
\hline & & Extracción de características en imágenes & 3 \\
\hline & & Series temporales y minería de flujos de datos & 3 \\
\hline & & $\begin{array}{l}\text { Sistemas de recuperación de información y de } \\
\text { recomendación }\end{array}$ & 3 \\
\hline & & Minería de datos: Aspectos avanzados & 3 \\
\hline & & $\begin{array}{l}\text { Modelos de ciencia de datos no numéricos. } \\
\text { Aplicaciones en redes sociales, web y gestión } \\
\text { de procesos }\end{array}$ & 6 \\
\hline & $\begin{array}{l}\text { Big data y } \\
\text { cloud } \\
\text { computing }\end{array}$ & Big data y cloud computing & 6 \\
\hline & \multirow{3}{*}{$\begin{array}{l}\text { Tecnologías } \\
\text { inteligentes e } \\
\text { inteligencia } \\
\text { computacional }\end{array}$} & Soft computing: Conjuntos y sistemas difusos & 4 \\
\hline & & $\begin{array}{l}\text { Técnicas de Soft Computing para Aprendizaje } \\
\text { y optimización. Redes Neuronales y } \\
\text { Metaheurísticas, programación evolutiva y } \\
\text { bioinspirada }\end{array}$ & 3 \\
\hline & & Visión por Computador & 3 \\
\hline & $\begin{array}{l}\text { Aplicaciones } \\
\text { de Ciencias de } \\
\text { Datos y } \\
\text { Tecnologías } \\
\text { Inteligentes } \\
\end{array}$ & $\begin{array}{l}\text { Aplicaciones de ciencia de datos y tecnologías } \\
\text { inteligentes }\end{array}$ & 6 \\
\hline
\end{tabular}


En la especialidad en Ciencia de Datos y Tecnologías Inteligentes es relevante la participación de los estudiantes en competiciones propias del máster y organizadas mediante la plataforma Kaggle [25]. La experiencia adquirida por los estudiantes ha permitido que, de forma particular, se hayan involucrado en competiciones profesionales encargadas por empresas y en las que se remunera a los ganadores. Los estudiantes del máster han trabajado con $\mathrm{R}$ [26], uno de los lenguajes más usados en ciencia de datos y con más de 6700 librerías disponibles en el repositorio CRAN (Comprehensive $R$ Archive Network). También cabe destacar que en este perfil los estudiantes tienen acceso a un clúster propio (hadoop.ugr.es) para la realización de las prácticas de Cloud Computing y Big Data, procesando los datos bajo los paradigmas Hadoop y Spark.

Por su parte, en la especialidad de Ingeniería de Computadores y Redes se pueden destacar por una parte las asignaturas relacionadas con la computación de altas prestaciones (HPC), implementando aplicaciones reales que maximicen el rendimiento de los sistemas de cómputo y analizando las características de éstos. Por otro lado, desde el bloque de asignaturas relacionado con los sistemas de aplicación específica, se estudia y trabaja de forma directa con sistemas que precisan de ingeniería y desarrollo a medida, tanto a nivel de hardware como de software. Los estudiantes podrán programar e implementar sus propios robots, actuadores y sistemas de control, diseñar sistemas de visión artificial o controlar un vehículo aéreo no tripulado, entre muchas otras aplicaciones.

Los detalles sobre los docentes que imparten cada materia, sus contenidos, objetivos, metodología docente, procedimiento de evaluación e información ampliada se pueden encontrar en la guía docente de cada asignatura. Estas guías de pueden consultar a través de la página web del Máster en Ciencia de Datos e Ingeniería de Computadores, en la sección "Descripción detallada del programa de estudios": http://masteres.ugr.es/datcom/pages/info academica/plan estudios.

En cuanto al calendario de impartición de las lecciones presenciales, éste comprende desde mediados del mes de octubre hasta final de mayo. Se ha procurado conciliar el posible desempeño de una actividad laboral por parte de los estudiantes, concentrando la docencia en horario de tarde y preferentemente de lunes a jueves. Además se incide en el carácter aplicado del máster, reservando un $50 \%$ de las lecciones presenciales para aplicaciones prácticas. Referente a la evaluación, aunque cada materia puede establecer sus propios matices y criterios, se propone un modelo basado en la evaluación continua, según lo descrito en la Normativa de evaluación y de calificación de los estudiantes de la Universidad de Granada [27].

\section{Conclusiones}

La Universidad de Granada ofrece la posibilidad de realizar la formación de posgrado en una de las universidades con mayor prestigio a nivel mundial en el sector de las TIC. Además, el Máster en Ciencia de Datos e Ingeniería de Computadores que se describe en esta contribución supone una oferta única que integra dos especialidades de gran interés social y en $\mathrm{I}+\mathrm{D}+\mathrm{i}$ : la ciencia de datos y la ingeniería de computadores. 
Los alumnos que superen este máster obtienen un título oficial con reconocimiento en todo el Espacio Europeo de Educación Superior (EEES).

Este máster se propone para su realización en un curso académico y combina formación rigurosa en técnicas avanzadas con un contacto muy cercano con aplicaciones reales y con una plantilla docente altamente cualificada. En definitiva, el Máster en Ciencia de Datos e Ingeniería de Computadores apuesta por la calidad y la excelencia en la formación de un perfil con una enorme proyección laboral e investigadora en la actualidad.

\section{Referencias}

1. Universidad de Granada, Máster Universitario Oficial en Ciencia de Datos e Ingeniería de Computadores, http://masteres.ugr.es/datcom/

2. Universidad de Granada, Programa Oficial de Doctorado en Tecnologías de la Información y la Comunicación, http://doctorados.ugr.es/tic/

3. Consejo de Gobierno de la Universidad de Granada: Normativa de Órganos Colegiados: NCG80/2. Máster Universitario en Ciencia de Datos e Ingeniería de Computadores. Boletín Oficial de la Universidad de Granada, no80. 11 de abril de 2014. (2014)

4. Consejería de Economía, Innovación, Ciencia y Empleo, Junta de Andalucía: Decreto 113/2014, de 15 de julio. Boletín Oficial de la Junta de Andalucía, Boletín número 138 de 17/07/2014 (2014)

5. Ministerio de Educación, Cultura y Deporte, Gobierno de España: Resolución de 2 de octubre de 2014, de la Secretaría General de Universidades, por la que se publica el Acuerdo del Consejo de Ministros de 26 de septiembre de 2014, por el que se establece el carácter oficial de determinados títulos de Máster y su inscripción en el Registro de Universidades, Centros y Títulos., Boletín Oficial del Estado, núm. 253 de 18 de octubre de 2014, páginas 84664 a 84675 (2014)

6. Universidad de Granada: Resolución de 25 de noviembre de 2014, de la Universidad de Granada, por la que se publica el plan de estudios de Máster en Ciencia de Datos e Ingeniería de Computadores., Boletín Oficial del Estado, núm. 304, de 17 de diciembre de 2014, páginas 102247 a 102249 (2014)

7. Universidad de Granada, Máster Universitario en Desarrollo de Software, http://masteres.ugr.es/master-desarrollo-software/

8. Universidad de Granada, Máster Universitario en Gestión y Tecnologías de Procesos de Negocio, http://masteres.ugr.es/mbagestiontic/

9. Universidad de Granada, Máster Universitario en Ingeniería de Telecomunicación, http://masteres.ugr.es/telecomunicacion/

10.Universidad de Granada, Máster Universitario en Ingeniería Informática, http://escuelaposgrado.ugr.es/static/EP Management/*/showCard/M50/56/2

11.El Confidencial, España necesita medio millón de expertos en informática más de los que puede aportar, http://www.elconfidencial.com/espana/2015-04-17/espana-necesita-mediomillon-de-expertos-en-informatica-mas-de-los-que-puede-aportar 761546/

12.Ediciones Cinco Días, El 'big data' arrasa en las escuelas de negocios, http://cincodias.com/cincodias/2015/05/01/sentidos/1430493583 087196.html

13.Universia España, El científico de datos: una novedosa y necesaria profesión, http://noticias.universia.es/ciencia-nn-tt/noticia/2014/05/06/1095994/cientifico-datosnovedosa-necesaria-profesion.html

14.Valdivia, J.F.: Las TIC: el nuevo motor económico de Granada. Diario IDEAL, Granada $12 / 4 / 2015,33(2015)$ 
15.Center for World-Class Universities (CWCU) of Shanghai Jiao Tong University,, 2014 Academic Ranking of World Universities (ARWU), http://www.shanghairanking.com/es/

16.National Taiwan University, 2014 National Taiwan University Ranking (NTU Ranking), http://nturanking.lis.ntu.edu.tw/

17.Duranton, M., Bosschere, K.D., Cohen, A., Maebe, J., Munk, H.: HiPEAC Vision 2015. High Performance and Embedded Architecture and Compilation. In: FP7 HiPEAC Network of Excellence (ed.), http://www.hipeac.org/assets/public/publications/vision/hipeac-vision2015 Dq0boL8.pdf

(2015)

18.Fernández de Vega, F., Hidalgo Pérez, J.I., Lanchares, J.: Parallel Architectures and Bioinspired Algorithms. Studies in Computational Intelligence, pp. 286. Springer, Berlin (2012)

19.Hwang, K., Fox, G.C., Dongarra, J.J.: Distributed and cloud computing : from parallel processing to the Internet of things. Elsevier Morgan Kaufmann, Waltham [Massachusetts] (2012)

20.Mayer-Schönberger, V., Cukier, K., Iriarte, A.: Big data : la revolución de los datos masivos. Turner, Madrid (2013)

21.Provost, F., Fawcett, T.: Data science for business : what you need to know about data minig and data-analytic thinking. O'Reilly, Sebastopol, CA (2013)

22.Leskovec, J., Rajaraman, A., Ullman, J.D.: Mining of massive datasets. Cambridge University Press, Cambridge (2014)

23.Schutt, R., O'Neil, C.: Doing data science : straight talk from the frontline. O'Reilly, Sebastopol [California] (2014)

24.Herrera, F.: Big data: Procesando los datos en la sociedad digital. Revista española de Física 28, 40-44 (2014)

25.Kaggle Community, Kaggle: The Home of Data Science, http://www.kaggle.com

26.R Foundation, R: The R Project for Statistical Computing, http://www.r-project.org/

27. Consejo de Gobierno de la Universidad de Granada, Normativa de evaluación y de calificación de los estudiantes de la Universidad de Granada, http://secretariageneral.ugr.es/pages/normativa/fichasugr/ncg7121 